\title{
Genome-wide sequencing reveals microRNAs downregulated in Cerebral Cavernous Malformations
}

Souvik Kar ${ }^{1, *}$, Kiran Kumar Bali ${ }^{2}$, Arpita Baisantry ${ }^{3}$, Robert Geffers ${ }^{4}$, Helmut Bertalanffy ${ }^{1}$

${ }^{1}$ International Neuroscience Institute, Rudolf-Pichlmayr-Strasse 4, D-30625, Hannover, Germany,

${ }^{2}$ Pharmacology Institute, Medical Faculty Heidelberg, Heidelberg University, Heidelberg, Germany

${ }^{3}$ Department of Kidney, Liver and Metabolic Diseases, Children's Hospital, Hannover Medical School, Hannover, Germany

${ }^{4}$ Genome Analytics Research Group, Helmholtz Centre for Infection Research, Braunschweig, Germany

\section{*Corresponding author:}

Souvik Kar, Ph.D,

International Neuroscience Institute,

Rudolf-Pichlmayr-Straße 4, D-30625,

Hannover, Germany

Tel: +49 511 27092-714.

E-mail: kar@ini-hannover.de 


\begin{abstract}
Cerebral cavernous malformations (CCM) are vascular lesions associated with loss of function mutations in one of the three genes encoding KRIT1 (CCM1), CCM2 and PDCD10. Recent understanding of the molecular mechanisms that lead to CCM development is limited. The role of microRNAs (miRNAs) has been demonstrated in vascular pathologies resulting in loss of tight junction proteins, increased vascular permeability and endothelial cell dysfunction. Since the relevance of miRNAs in CCM pathophysiology has not been elucidated, the primary aim of the study was to identify miRNA-mRNA expression network associated with CCM. Using small RNA sequencing, we identified a total of 764 matured miRNAs expressed in CCM patients compared to the healthy brains. The expression of the selected miRNAs was validated by qRT-PCR and the results were found to be consistent with the sequencing data. Upon application of additional statistical stringency, five miRNAs (let-7b-5p, miR-361-5p, miR-370-3p, miR-181a-2-3p and miR-95-3p) were prioritized to be top CCM-relevant miRNAs. Further in silico analyses revealed that the prioritized miRNAs have direct functional relation with mRNAs, such as MIB1, HIF1A, PDCD10, TJP1, OCLN, HES1, MAPK1, VEGFA, EGFL7, NF1 and ENG, which are previously characterized as key regulators of CCM pathology. To date this is the first study to investigate the role of miRNAs in CCM pathology. By employing cutting edge molecular and in silico analyses on clinical samples, the current study reports global miRNAs expression changes in CCM patients and provides a rich source of data set to understand detailed molecular machinery involved in CCM pathophysiology.
\end{abstract}

Keywords: Cerebral cavernous malformations; microRNAs; vascular; pathology; in silico analyses; sequencing. 


\section{Introduction}

Cerebral cavernous malformations (CCM) are neurovascular lesions affecting approximately $0.5 \%$ of the general population (Fisher et al., 2013; Kar et al., 2016). The lesions comprise of dilated capillary vessels without intervening brain parenchyma and symptoms include severe headaches, focal neurological deficits and seizures (Bertalanffy et al., 1992; Gingras et al., 2012; Samii et al., 2001; Schulz et al., 2015) (Fig. 1). CCM may occur either as sporadic or as familial forms. The familial cases consist of autosomal-dominantly inherited forms with incomplete clinical penetrance (Fisher et al., 2014; Hwang et al., 2014; Kar et al., 2015). A majority of inherited forms of CCM follows a "second hit" or a Knudsonian mutation, where loss of one allele due to a germline mutation in an affected cell (first hit) is succeeded by a somatic mutation in the other (second hit) (Akers et al., 2009). So far three types of mutations have been identified in the CCM causing genes: KRIT1 (CCM1), CCM2 (OSM or malcavernin) and PDCD10 (CCM3) and the products of these genes have been predicted to be involved in a common functional pathway (Draheim et al., 2014). A recent hypothesis regarding the existence of a fourth unidentified CCM gene was also proposed to explain the low frequency of mutation identified in families within the PDCD10 (10\%) at the CCM3 locus (Riant et al., 2010). Despite recent advances in understanding the structure and function of these proteins, much remains to be known how the loss of such proteins leads to CCM pathogenesis.

MicroRNAs (miRNAs) represent a class of short, non-coding RNAs, about $20 \sim 22$ nucleotides in length that post-transcriptionally regulate gene expression. During its biogenesis, the mature strand incorporates into the RNA-induced silencing complex (RISC) and results in translational repression by binding to the complementary sequences of the messenger RNAs (Kala et al., 2013). MiRNAs have been implicated in diverse biological activities including: tumorigenesis (Dong et al., 2010), hematopoiesis (Chen et al., 2004), epigenetics (Dong et al., 2010) and angiogenesis (Suarez et al., 2009; Yin et al., 2015). MiRNAs that are involved in the process of angiogenesis are classified as "angiomiRs" and a plethora of angiomiRs have been recently identified across various animal models, including mice, zebrafish and humans (Wang et al., 2013). For instance, miR-126 is highly enriched in the endothelial cells and has an essential role in the blood vessel formation and integrity (Lopez-Ramirez et al., 2016; Wang et al., 2008). Another potent angiomiR, miR-210 is a well-known mediator of angiogenesis and has been found to be significantly up-regulated following cerebral ischemia in rats (Lou et al., 2012). Although there are studies reporting the role of miRNAs in angiogenesis (Chen et al., 2014; Seok et al., 2014; Suarez et al., 2009; Wang et al., 2013), in the pathogenesis of ischemic brain injury (Li et al., 2016; 
Liu et al., 2013; Tan et al., 2009; Yin et al., 2010) and in cerebral arteriovenous malformation (AVM) (Ferreira et al., 2014), their relevance to CCM biology has been so far not reported.

Using genome-wide miRNA sequencing, we aimed to identify miRNAs expression profile in CCM patients compared with healthy brains. Applying highest level of statistical stringency resulted in candidate miRNAs significantly downregulated in CCM. After shortlisting the prioritized miRNAs, we sought to identify their target genes associated with CCM. Our search represented notable enrichment of CCM-relevant genes. Identification of genes targeted by these candidate miRNAs might help to understand their mechanism of regulation during CCM development and might, therefore aid in developing future therapeutic targets.

\section{Methods}

\section{Ethics Statement}

The study was performed in accordance with the guidelines and with permission by the Ethical Committee of the Hannover Medical School, Germany (Approval Number 6960).

\section{Patient sample collection}

Brainstem biopsies obtained from three patients (Cav 1, Cav2 and Cav3) operated due to cavernous malformations were snap frozen in liquid nitrogen. The samples were stored temporarily at $-80^{\circ} \mathrm{C}$ until total RNA extraction. CCM diagnosis was based on the MRI and histopathological criteria as described previously (Bertalanffy et al., 2002). Each patient had at least one or more clinical presentations manifested by seizures, hemorrhages and focal neurological deficits (Table 1). Additionally, three commercially available Human Brain Reference total RNA (Amsbio) (Con1, Con2 and Con3) pooled from healthy adult brain were used as corresponding controls.

\section{RNA isolation and integrity}

Tissue biopsies were stored in at least 10 volumes of RNAlater-ICE (AM7030; Ambion) at $-80^{\circ} \mathrm{C}$ prior to RNA extraction. The tissue was lysed using ceramic beads (Precellys system, CK14L; Peqlab) in $600 \mu 1$ of lysis buffer from mirVANA miRNA isolation Kit (AM1560; Ambion). Homogenization was performed using Precellys 24 Homogenizer (Peqlab) with 4 pulses of $5 \mathrm{sec}$ at $6000 \mathrm{rpm}$. Total RNA was extracted with the mirVANA Kit according to the recommended protocol variant for total RNA including small species. RNA was finally eluted with $100 \mu \mathrm{l}$ of elution solution preheated to $95^{\circ} \mathrm{C} .87,5 \mu \mathrm{l}$ of eluted RNA were subjected to an DNase-I treatment 
in solution for 10 minutes at RT by adding $10 \mu \mathrm{l}$ of buffer RDD and 2,5 $\mu$ l of DNase-I, both taken from RNeasy Micro Kit (74004; Qiagen). After DNase-I treatment $10 \mu \mathrm{l}$ of $5 \mathrm{M} \mathrm{NaCl}$ and $152 \mu 1$ of $100 \%$ Ethanol were added, all was thoroughly mixed and loaded onto an RNeasy column for final purification according to the standard RNeasy procedure, except that buffer RW1 was diluted 1:3 with 100\% Ethanol prior to usage (in order to enable binding of even small RNA species to the silica matrix). Total RNA was finally eluted with $14 \mu$ l of RNase free water. RNA was quantified and purity was determined with the Nanodrop ND-1000 spectrophotometer (Peqlab). RNA integrity was determined with an Agilent Bioanalyzer 2100 using the RNA 6000-Nano assay (5067-1511; Agilent Technologies). RNA integrity numbers (RINs) ranged from 7.7 to 8.3 for the RNA samples purified from CCM tissues and 8 to 9 for the three normal human brain tissues.

\section{Library preparation and sequencing}

The RNA sequencing library was generated from 50-100ng total RNA using TruSeq® Small RNA Library Prep Kits v2 (Illumina) according to manufacturer's protocols. The libraries were sequenced on Illumina HiSeq2500 using TruSeq SBS Kit v3-HS (50 cycles, single ended run) with an average of $10 \times 10^{6}$ reads per RNA sample.

\section{Bioinformatics and data analysis}

Raw data (FASTQ files) were applied to OASIS 1.0 sRNA Detection pipeline (Capece et al., 2015). Briefly, FASTQ files were trimmed with cutadapt 1.7.1 (http://code.google.com/p/cutadapt/) removing Illumina adapter sequences (TGGAATTCTCGGGTGCCAAGG) and nucleotides with phred scores below 20. The minimummaximum limit for sequence length was set to 15-32 nucleotides. Alignment of trimmed FASTQ sequences was done with STAR Aligner (Dobin et al., 2013) against the following databases: Mirbase version 20; release date: 2013-10-1, snRNA: Ensembl: Human s. version GRCh38.74; snoRNAEnsembl: Human s. version GRCh38.74; rRNA: Ensembl: Human s. version GRCh38.74. The number of mismatches allowed for successful alignment was set to $5 \%$ of sequence length. Counts per small RNA (feature) were calculated using feature Counts (Liao et al., 2014). Differential expression of small RNA was determined by DESeq2 (Love et al., 2014).

\section{Differential expression}

Following alignment, the read counts of each small RNA element were converted to "reads per million" (RPM) using the formula: normalized expression $=$ reads assigned to a unique sequence in a sample/total number of reads identified by all sequences in that sample x 1,000,000 (Motameny et al., 2010). The matured miRNAs were then filtered from other small non-coding RNAs (novelmiRNAs, snoRNAs, piRNAs and rRNAs). This 
yielded a total of 764 matured miRNA sequences. Next, we applied an arbitrary filter of RPM values $\geq 30$ in at least two out of 6 tested samples. This came down to 327 differentially expressed miRNAs in CCM compared to the control samples.

The differential expression of the individual miRNAs was calculated based on the degree to which the RPM values were up- or downregulated in CCM with respect to the control samples. This was statistically evaluated as follows: For each miRNAs in each of the Cavernoma pools ( $\mathrm{Cav} 1$, Cav 2 and Cav 3) we calculated differential regulation with respect to each of the Control (Con 1, Con 2 and Con 3) pools (Cav 1/Con 1), (Cav1/Con 2), (Cav 1/Con 3), (Cav 2/Con 1), (Cav 2/Con 2), (Cav 2/Con 3) and (Cav 3/Con 1), (Cav 3/Con 2), (Cav 3/Con 3). The result was 9 estimates of differentially regulated CCM with a corresponding mean \pm standard error of the mean (SEM). The same was done for calculating the average fold-difference calculated from normalized RPM values. Subsequent consistency in terms of biological stability was achieved by application of BenjaminiHochberg False Discovery Rate (FDR) and Bonferroni FDR correction method.

\section{qPCR validation of miRNAs}

To validate the sequencing data, we randomly selected three miRNAs downregulated in CCM tissue samples with highest statistical stringency. 40 ng of total RNA was reverse transcribed with miRNA-specific RT primers using the $\operatorname{TaqMan}^{\circledR}$ MicroRNA Reverse Transcription Kit (Applied Biosystems, \#4366597) following manufacturer's protocol. Using miRNA-specific primers and TaqMan ${ }^{\circledR}$ Universal Master Mix II-no UNG (Applied Biosystems, \#4440040) the cDNA template was amplified with the help of StepOnePlus ${ }^{\text {TM }}$ Real-Time PCR (Applied Biosystems, Germany). The assay numbers to validate miRNAs are as follows: hsa-let-7b-5p (002619), hsa-miR-181a-2-3p (002317) and hsa-miR-95-3p (000433). Expression levels of the target miRNAs were normalized to that of RNU6B (Assay number: 001093) and the fold change was determined by $2^{-\Delta \Delta C t}$. All reactions were run in triplicate.

\section{Prediction of putative and validated gene targets}

Target prediction for the five downregulated miRNAs was determined in silico using miRWalk 2.0 algorithm. Briefly, the selected miRNAs were uploaded on the "Predicted and Validated Target Module" of miRWalk 2.0 search option and the subsequent list of putative and experimentally-validated targets relative to each prioritized miRNAs were obtained. These were further scanned and analyzed for Gene Ontology Molecular Function (GOMF) enrichment terms and Koyoto Encyclopedia of Genes and Genomes (KEGG) pathways classification. Network analysis for miRNAs-mRNA putative target interaction was carried out using Cytoscape 3.3.0. 


\section{Statistical analysis}

All data are expressed as mean \pm standard error of the mean (SEM). The One-way ANOVA test was used to calculate the significance of differential expression level for each miRNAs between CCM and normal brain. Heatmap for the 52 significantly differentially regulated miRNAs were plotted using freely available online software, matrix2png (Pavlidis et al., 2003). For the estimation of significant miRNAs, Benjamini-Hochberg False Discovery Rate (FDR) correction (adjusted p-values) (Benjamini et al., 1995) were applied. For extra confidence, Bonferroni multiple-comparison correction method (adjusted p-values) was implemented to assess the statistically significant miRNAs. Corrected $\mathrm{p} \leq 0.05$ were considered to be statistically significant.

\section{Results}

\section{miRNA sequencing in CCM patients}

Procurement of fresh healthy human brain tissue has always been a challenging task. Also, a major practical concern during microsurgical resection lies in delineating adjacent healthy brain tissue from the CCM lesion. On the other hand, Amsbio Human Brain Reference total RNA pooled from healthy individuals is a robust, thoroughly characterized commercially available human tissue RNA widely used as a standard reference for studying changes in miRNA expression.

Total RNA isolated from CCM patients $(n=3)$ and commercially available FirstChoice ${ }^{\circledR}$ Human Brain Reference RNA ( $n=3$ ) were sequenced for small RNAs. After trimming of FASTAQ files and removal of the adapter sequences, 16 to 23 million read sequences belonging to each sample were mapped to the human genome, with a total of 118.9 million reads. The minimum and maximum read length was between 15-32 nucleotide sequences and the average read length for six libraries were: Con1: 21.50; Con2: 21.57; Con3: 21.65; Cav1: 21.59; Cav2: 21.82 and Cav3: 21.82 base pairs respectively.

MiRBase-v20 recognizes a total of 2555 mature human miRNA sequences. Of these annotated mature miRNAs, 764 were identified to be expressed in the six libraries sequenced. The abundances of each small RNA was normalized using "reads per million (RPM)" in each small RNA library (see Methods) which ranged from several hundreds to millions. For example, in the Cav1 library, 55.23\% miRNAs were low expressed $(<10$ RPM), $43.45 \%$ were moderately expressed (10-10,000 RPM) and only $1.32 \%$ of them were abundantly expressed (>10,000 RPM) (Fig. 2). In order to identify the miRNAs that were stably expressed across biological samples, we applied an arbitrary filter having RPM values $\geq 30$ in at least two out of 6 tested samples as 
previously described (Bali et al., 2013). Applying such filter resulted in 327 miRNAs with stable expression and abundance across CCM tissues. A pairwise comparison of the 327 expressed miRNAs between CCM and healthy brain is shown in the volcano plot (Fig. 3).

\section{Differential regulation of miRNAs in CCM}

Following identifying a set of stably expressed miRNAs in healthy and CCM tissue samples, we investigated the magnitude and significance of changes in miRNA expression between the two groups. For this, we first calculated the fold-change in the expression of each of 327 miRNAs in the CCM samples as compared to healthy brain samples (as explained in the methods section). Applying statistical P-value cut-off of 0.05 revealed that expression of 162 miRNAs was significantly different in CCM tissue samples. In the next steps, we calculated the adjusted P value (adjP) by applying Benjamini-Hochberg False Discovery Rate (FDR) correction for highthroughput experiments and identified that 52 miRNAs were regulated in CCM with high statistical confidence in comparison to healthy brain samples. The results of these 52 significantly regulated miRNAs are summarized as Additional file 1: Table S1. Among them, 10 were significantly up-regulated with miR-27a-5p having the highest fold-change $(79.78$, padj $\leq 0.05)$ followed by miR-21-5p $(67.99$, padj $\leq 0.05)$, miR-146b-5p $(24.08$, padj $\leq 0.05), \operatorname{miR}-155-5 p(19.88, \operatorname{padj} \leq 0.05), \operatorname{miR}-210-3 p(15.7, \operatorname{padj} \leq 0.05), \operatorname{miR}-214-3 p(11.02, \operatorname{padj} \leq 0.05)$, $\operatorname{miR}-493-3 p(9.04$, padj $\leq 0.05)$, miR-34a-5p (8.95, padj $\leq 0.05), \operatorname{miR}-301 b(6.95, p a d j \leq 0.05)$ and $\operatorname{miR}-22-5 p$ (3.69, padj $\leq 0.05)$, respectively. In addition to the up-regulated miRNAs, 42 miRNAs were downregulated in CCM tissues $(p \leq 0.05)$ with miR-128-3p having the lowest fold-change $(0.007, \operatorname{pdj} \leq 0.05)($ Fig. 4$)$.

In the next steps, we set out to identify most biologically significant miRNAs from above identified 52 CCMrelevant miRNAs. For this, we applied increasing levels of statistical stringency (Bali et al., 2014) for FDR correction. Executing Bonferroni-step down or Bonferroni FDR consistently identified five miRNAs (miR-953p, miR-370-3p, let-7b-5p, miR-181a-2-3p and miR-361-5p) to be significantly downregulated in CCM with highest statistical stringency. This ensured extra confidence in terms of biological stability across the libraries. The miR-95-3p had the lowest fold-change $(0.09$, padj $\leq 0.05)$ followed by miR-370-3p $(0.12$, padj $\leq 0.05)$, let7b-5p $(0.17$, padj $\leq 0.05)$, miR-181a-2-3p $(0.26$, padj $\leq 0.05)$ and miR-361-5p $(0.36$, padj $\leq 0.05)$, respectively. A brief overview of the subsequent steps applied with increasing levels of stringency is shown in Table 2.

\section{Validation by qRT-PCR assay}

To further validate the miRNA expression screen with an independent qRT-PCR method, we randomly chose three differentially regulated miRNAs among 52 CCM-relevant miRNAs with highest statistical stringency. 
Analyzing the qRT-PCR data obtained by using specific probes for let-7b-5p, miR-181a-2-3p and miR-95-3p revealed that their expression is significantly lesser in CCM tissue samples as compared to control samples (Fig. 5). These observations confirm that the change in the expression of the tested miRNAs is consistent with observations from the preliminary miRNA sequencing data.

\section{Bioinformatics analysis of the validated miRNAs}

\section{Putative target gene prediction}

After identifying and validating CCM-relevant miRNAs, we sought to understand the mechanisms of action of these miRNAs. For this, we first determined putative mRNAs for each of the five miRNAs (let-7b-5p, miR-3615p, miR-370-3p, miR-181a-2-3p and miR-95-3p) by employing miRWalk 2.0 algorithm. miRWalk 2.0 provides a comprehensive list of putative and validated mRNAs for a given miRNA by combining results from multiple target prediction algorithms (Dweep et al., 2011). To augment inter sample consistency within our data, we applied seven different target prediction algorithms (miRWalk, MicroT4, miRanda, miRDB, Pictar2, PITA and Targetscan) for the identification of putative miRNAs targets.

Our in silico analysis revealed multiple mRNAs for each miRNAs individually screened. The total number of high-confidence mRNA hits generated by let-7b-5p, miR-361-5p, miR-370-3p, miR-181a-2-3p and miR-95-3p were 726, 578, 449, 101 and 23, respectively (Additional file 2: Table S2). The search for the CCM-relevant mRNAs targeted by the five prioritized miRNAs was done manually by visual inspection and by considering CCM-relevant knowledge about each target into account. This resulted in the identification of CCM-relevant mRNAs targeted by let-7b-5p, miR-361-5p and miR-370-3p, while miR-181a-2-3p and miR-95-3p had none (Fig. 6).

We then extended our study to determine and ranked the miRNA-mRNA putative target interaction by generating a network using Cytoscape 3.3.0 (Shannon et al., 2003) Fig. 6. As shown in the interaction map, the green boxes are CCM-relevant mRNAs targeted individually by miR-361-5p, miR-370-3p and let-7b-5p. No CCM-specific mRNAs were targeted by miR-181a-2-3p and miR-95-3p.

To characterize miRNAs in terms of Molecular Function and for determining the underlying biological functions we extended our approach to define which Gene Ontology Molecular Function (GOMF) terms and Koyoto Encyclopedia of Genes and Genomes (KEGG) pathways were enriched by the downregulated miRNAs. The GOMF enrichment results showed that zinc ion and ATP binding were significantly enriched (FDR corrected p- 
value of $\leq 0.05$ ) while KEGG pathway analysis revealed Pathways in cancer as the most enriched pathways with a FDR corrected p-value of $\leq 0.05$. It is noteworthy to mention that "Pathways in cancer" is a combination of several pathways, including pathways reported to play a key functional role in CCM pathology (VEGF, PI3K/Akt and MAPK signaling), indicating that several vital processes might be regulated by these miRNAs.

\section{Validated target gene prediction}

Validated or experimentally verified target mRNA prediction for each of the five downregulated miRNAs was explored in silico using miRWalk 2.0 algorithm (Dweep et al., 2011). Our analysis revealed 981 mRNA hits targeted by let-7b-5p, 93 by miR-361-5p, 4 mRNAs by miR-370-3p and one mRNA each by miR-95-3p and miR-181a-2-3p. As mentioned above in 'putative target gene prediction', we applied similar approach for the identification of CCM-relevant mRNAs targeted by the selected miRNAs. These resulted in mRNAs: MIBI, HIF1A, PDCD10, TJP1, OCLN, HES1, MAPK1 and ENG targeted by let-7b-5p which code for mindbomb E3 ubiquitin protein ligase 1 (Sure et al., 2005), hypoxia-inducible factor 1-alpha (Sure et al., 2005), programmed cell death 10 (Bergametti et al., 1995; Liquori et al., 2007), tight junction protein1 (Jakimovski et al., 2014; Schneider et al., 2011), occludin (Schneider et al., 2011), hes family bHLH transcription factor 1 (You et al., 2013), mitogen-activated protein kinase 1 (Wustehube et al., 2010]) and endoglin (Sure et al., 2005), respectively. Additionally, miR-361-5p targeted VEGFA (You et al., 2013) and EGFL7 (Renz et al., 2015) while miR-370-3p targeted NF1 (Rerat et al., 2015) mRNAs respectively. The subsequent results are summarized in Table 3.

\section{Discussion}

$\mathrm{CCM}$ in the brainstem region are rare, deep seated lesions having a higher propensity of bleeding. If left untreated, it may even lead to death of the patient. With the advent of improved diagnostic tools and the advancement of magnetic resonance imaging (MRI), there has been a significant progress in the early clinical diagnosis of brainstem CCM (Bozinov et al., 2010). Currently, the only therapeutic option for the treatment of deep seated CCM is microsurgical resection. Therefore, it is necessary to identify potential biomarkers that may open up new avenues for alternative therapeutic approach. The role of miRNAs in regulating angiogenesis and in stroke pathogenesis has been reported (Kuehbacher et al., 2008; Liu et al., 2011; Wang et al., 2008; Yin et al., 2015; Yuan et al., 2016), however their relevance to brain vascular malformations notably, CCM have not been so far elucidated. Here, we report for the first time genome-wide miRNA expression profiling in CCM patients and believe our dataset will be valuable for future research to discern the role of miRNAs in CCM pathology. 
Using a comprehensive genome-wide sequencing approach, we identified a total of 764 miRNAs expressed in CCM. By implementing in-depth statistical analysis, we identified 10 up-regulated and 42 downregulated miRNAs in the CCM patients. By further applying increasingly stringent statistical methods, we identified 5 miRNAs namely let-7b-5p, miR-361-5p, miR-370-3p, miR-181a-2-3p and miR-95-3p significantly downregulated in CCM.

A key factor in identifying the function of miRNAs is through their putative mRNAs. With the advent of bioinformatics and multiple target prediction algorithms, it has been possible to explore the function of miRNAs and establish their biological significance in diseases. A plethora of algorithms and databases have been developed based on pairing strategies between miRNA and mRNA (Khan et al., 2015). The current available target prediction algorithms are believed to contain a high degree of false-positive rates. Therefore, in order to circumvent false positives and increase the confidentiality and credibility of true predictions, we applied seven established target prediction algorithms using web based miRWalk 2.0 (see Results) (Dweep et al., 2011). Our search yielded few hundreds each of putative mRNAs for let-7b-5p, miR-361-5p, miR-370-3p, miR-181a-2-3p and miR-95-3p. Most striking finding was that each of the prioritized miRNAs are predicted to target mRNAs which were previously determined to play a key functional role in CCM pathology. For example, several genes, such as MLLT4, VEGFA, MAPK1, RAC1, RHOA, FOXO1, ENG, SMURF1 and HEYL (Draheim et al., 2014; Glading et al., 2007; Goitre et al., 2010; Schulz et al., 2015; Sure et al., 2005; Wustehube et al., 2010; You et al., 2013; Zawistowski et al., 2005; Zheng et al., 2010) were identified to have binding sites for one of the 3 prioritized miRNAs from our screen namely let-7b-5p, miR-361-5p and miR-370-3p (Fig. 6), suggesting functional validity and relevance of the screen we performed.

Another interesting finding was that several of CCM-relevant mRNAs are previously characterized to be functional targets of our 3 prioritized miRNAs in distinct experimental systems. In other words, miRNAs prioritized from our genome-wide screen are known to functionally target several key CCM-relevant mRNAs. For example, miR-361-5p is known to modulate the expression of $V E G F$, a key gene involved in CCM pathophysiology, in diseased endothelial progenitor cells (EPC) in vitro (Wang et al., 2014). In another recent study, let-7, known as hypoxia-responsive miRNA, is robustly induced by HIF1A in the vascular endothelial cells (Chen et al., 2013). Despite previous studies reporting the role of miRNA-mRNA in vascular endothelial cells, their mechanism of regulation in CCM would require further study.

Another critical point of interest is the mechanisms via which miRNAs function in the context of CCM. Since all 5 prioritized miRNAs were downregulated in cavernoma conditions, according to existing understanding of 
miRNA mediated mechanisms, they are expected to predominantly target mRNAs which accelerate the cavernoma progression. Sure et al studied the proliferative activity of the endothelium in $56 \mathrm{CCM}$ patients and showed increased expression of VEGF, MIB1, HIF1 and ENG in endothelial cells (Sure et al., 2005). On the other hand, there are genes such as TJP1, OCLN, HES1 which are known to be downregulated in CCM conditions (Schneider et al., 2011; You et al., 2013) but also have binding sites for our prioritized miRNAs, which makes it difficult to explain their function with the existing model of miRNA function. However, it has to be noted that the screen represents the whole cavernoma tissue, which include neuronal and non-neuronal cells and the phenotype depends of the cell-type specific expression of a given miRNA and its target gene. Therefore, it would be intriguing to determine cell-type specific mechanism interaction between prioritized miRNAs and putative/validated targets in the context of CCM. Future studies should warrant the understanding of whether miRNA-mRNA interactions follow classical regulatory pattern in CCM pathology. Additionally, although we concentrated on only 5 miRNAs with strongest statistical significance, there were also 47 other miRNAs which provide an unlimited source of substrate for future studies with respect to CCM pathology.

An additional intriguing observation was the identification of several isoforms of same mRNA to be targeted by single miRNA. Formation of mRNA isoforms by alternative splicing is one of the important cellular mechanisms for proteome diversity. Our results revealed that 18 different isoforms for vascular endothelial growth factor A (VEGFA) have putative binding sites for miR-361-5p. VEGF is known to play a significant role in the formation of new blood vessels and is crucial for the induction of proliferation and differentiation of endothelial cells. Aberrant regulation of VEGF-mediated signalling contributes to angiogenesis and severe brain pathologies. The role of VEGF in CCM pathology has been previously reported (He et al., 2010; Sure et al., 2005; You et al., 2013). Although the role of miR-361-5p and its target VEGFA has been previously reported in human cutaneous squamous cell carcinoma and in patients with coronary artery disease (Kanitz et al., 2012; Wang et al., 2014), their mechanism of interaction in CCM is yet to be established. Therefore, it would be extremely interesting to study functional regulation of VEGF isoforms with respect to miR-361-5p. Another observation was the identification of the two isoforms (rac1 and raclb) of ras-related $\mathrm{C} 3$ botulinum toxin substrate $1(R A C 1)$ mRNA targeted by miR-361-5p. Earlier studies have reported that CCM2 binds the mitogen-activated protein kinase (MAPK) kinase kinase MEKK3 and RAC1 to regulate the p38 MAPK cascade (Zawistowski et al., 2005). Besides, isoforms of 6 other mRNAs have putative binding sites for three of our prioritized miRNAs. Likewise for the mRNAs validated to be targets of the prioritized miRNAs, we identified that three isoforms of the gene, neuroblastoma RAS viral oncogene homolog (NRAS) are targeted by let-7b-5p. From the observations above, 
we hypothesize that let-7b-5p, miR-361-5p, and miR-370-3p could affect CCM pathology by modulating their mRNA isoforms generated due to alternative splicing.

\section{Conclusion}

This study provides the first evidence that there are candidate miRNAs crucial for CCM pathology. We conclude that let-7b-5p, miR-361-5p and miR-370-3p may be of biological and functional relevance for patients suffering from CCM within the brainstem. The identification of key CCM-relevant mRNAs as putative as well as validated targets for these three prioritized miRNAs strongly indicate their involvement in signaling pathways crucial to CCM pathophysiology. Further functional characterization of these miRNAs in large patient cohorts and animal experimental models would be valuable in determining how they regulate their target gene expression and may be pivotal in considering miRNA antagonism or overexpression as novel therapeutic intervention for improved diagnosis in CCM patients.

\section{Acknowledgement}

The authors are thankful to the Research Core Unit Transcriptomics (RCUT) of Hannover Medical School for their technical assistance and support with regard to RNA-isolation from brain tissue samples.

\section{Conflict of Interest}

The authors declare no conflict of interest.

\section{References}

1. Akers, A. L., E. Johnson, G. K. Steinberg, J. M. Zabramski and D. A. Marchuk (2009). "Biallelic somatic and germline mutations in cerebral cavernous malformations (CCMs): evidence for a two-hit mechanism of CCM pathogenesis." Hum Mol Genet 18(5): 919-930.

2. Bali, K. K., M. Hackenberg, A. Lubin, R. Kuner and M. Devor (2014). "Sources of individual variability: miRNAs that predispose to neuropathic pain identified using genome-wide sequencing." Mol Pain 10: 22.

3. Bali, K. K., D. Selvaraj, V. P. Satagopam, J. Lu, R. Schneider and R. Kuner (2013). "Genome-wide identification and functional analyses of microRNA signatures associated with cancer pain." EMBO Mol Med 5(11): 1740-1758.

4. Benjamini, Y. and Y. Hochberg (1995). "Controlling the False Discovery Rate: A Practical and Powerful Approach to Multiple Testing." Journal of the Royal Statistical Society. Series B (Methodological) 57(1): 289-300.

5. Bergametti, F., C. Denier, P. Labauge, M. Arnoult, S. Boetto, M. Clanet, P. Coubes, B. Echenne, R. Ibrahim, B. Irthum, G. Jacquet, M. Lonjon, J. J. Moreau, J. P. Neau, F. Parker, M. Tremoulet, E. 
Tournier-Lasserve and N. Societe Francaise de (2005). "Mutations within the programmed cell death 10 gene cause cerebral cavernous malformations." Am J Hum Genet 76(1): 42-51.

6. Bertalanffy, H., L. Benes, T. Miyazawa, O. Alberti, A. M. Siegel and U. Sure (2002). "Cerebral cavernomas in the adult. Review of the literature and analysis of 72 surgically treated patients." Neurosurg Rev 25(1-2): 1-53; discussion 54-55.

7. Bertalanffy, H., G. Kuhn, R. Scheremet and W. Seeger (1992). "Indications for surgery and prognosis in patients with cerebral cavernous angiomas." Neurol Med Chir (Tokyo) 32(9): 659-666.

8. Bozinov, O., T. Hatano, J. Sarnthein, J. K. Burkhardt and H. Bertalanffy (2010). "Current clinical management of brainstem cavernomas." Swiss Med Wkly 140: w13120.

9. Capece, V., J. C. Garcia Vizcaino, R. Vidal, R. U. Rahman, T. Pena Centeno, O. Shomroni, I. Suberviola, A. Fischer and S. Bonn (2015). "Oasis: online analysis of small RNA deep sequencing data." Bioinformatics 31(13): 2205-2207.

10. Chen, C. Z., L. Li, H. F. Lodish and D. P. Bartel (2004). "MicroRNAs modulate hematopoietic lineage differentiation." Science 303(5654): 83-86.

11. Chen, J., P. Venkat, A. Zacharek and M. Chopp (2014). "Neurorestorative therapy for stroke." Front Hum Neurosci 8: 382.

12. Chen, Z., T. C. Lai, Y. H. Jan, F. M. Lin, W. C. Wang, H. Xiao, Y. T. Wang, W. Sun, X. Cui, Y. S. Li, T. Fang, H. Zhao, C. Padmanabhan, R. Sun, D. L. Wang, H. Jin, G. Y. Chau, H. D. Huang, M. Hsiao and J. Y. Shyy (2013). "Hypoxia-responsive miRNAs target argonaute 1 to promote angiogenesis." J Clin Invest 123(3): 1057-1067.

13. Dobin, A., C. A. Davis, F. Schlesinger, J. Drenkow, C. Zaleski, S. Jha, P. Batut, M. Chaisson and T. R. Gingeras (2013). "STAR: ultrafast universal RNA-seq aligner." Bioinformatics 29(1): 15-21.

14. Dong, H., H. Siu, L. Luo, X. Fang, L. Jin and M. Xiong (2010). "Investigation gene and microRNA expression in glioblastoma." BMC Genomics 11 Suppl 3: S16.

15. Draheim, K. M., O. S. Fisher, T. J. Boggon and D. A. Calderwood (2014). "Cerebral cavernous malformation proteins at a glance." J Cell Sci 127(Pt 4): 701-707.

16. Dweep, H., C. Sticht, P. Pandey and N. Gretz (2011). "miRWalk--database: prediction of possible miRNA binding sites by "walking" the genes of three genomes." J Biomed Inform 44(5): 839-847.

17. Ferreira, R., T. Santos, A. Amar, S. M. Tahara, T. C. Chen, S. L. Giannotta and F. M. Hofman (2014). "MicroRNA-18a improves human cerebral arteriovenous malformation endothelial cell function." Stroke 45(1): 293-297.

18. Fisher, O. S. and T. J. Boggon (2014). "Signaling pathways and the cerebral cavernous malformations proteins: lessons from structural biology." Cell Mol Life Sci 71(10): 1881-1892.

19. Fisher, O. S., R. Zhang, X. Li, J. W. Murphy, B. Demeler and T. J. Boggon (2013). "Structural studies of cerebral cavernous malformations 2 (CCM2) reveal a folded helical domain at its C-terminus." FEBS Lett 587(3): 272-277.

20. Gingras, A. R., J. J. Liu and M. H. Ginsberg (2012). "Structural basis of the junctional anchorage of the cerebral cavernous malformations complex." J Cell Biol 199(1): 39-48.

21. Glading, A., J. Han, R. A. Stockton and M. H. Ginsberg (2007). "KRIT-1/CCM1 is a Rap1 effector that regulates endothelial cell cell junctions." J Cell Biol 179(2): 247-254. 
22. Goitre, L., F. Balzac, S. Degani, P. Degan, S. Marchi, P. Pinton and S. F. Retta (2010). "KRIT1 regulates the homeostasis of intracellular reactive oxygen species." PLoS One 5(7): e11786.

23. He, Y., H. Zhang, L. Yu, M. Gunel, T. J. Boggon, H. Chen and W. Min (2010). "Stabilization of VEGFR2 signaling by cerebral cavernous malformation 3 is critical for vascular development." Sci Signal 3(116): ra26.

24. Hwang, J. and D. C. Pallas (2014). "STRIPAK complexes: structure, biological function, and involvement in human diseases." Int J Biochem Cell Biol 47: 118-148.

25. Jakimovski, D., H. Schneider, K. Frei, L. N. Kennes and H. Bertalanffy (2014). "Bleeding propensity of cavernous malformations: impact of tight junction alterations on the occurrence of overt hematoma." J Neurosurg 121(3): 613-620.

26. Kala, R., G. W. Peek, T. M. Hardy and T. O. Tollefsbol (2013). "MicroRNAs: an emerging science in cancer epigenetics." J Clin Bioinforma 3(1): 6.

27. Kanitz, A., J. Imig, P. J. Dziunycz, A. Primorac, A. Galgano, G. F. Hofbauer, A. P. Gerber and M. Detmar (2012). "The expression levels of microRNA-361-5p and its target VEGFA are inversely correlated in human cutaneous squamous cell carcinoma." PLoS One 7(11): e49568.

28. Kar, S., A. Baisantry, A. Nabavi and H. Bertalanffy (2016). "Role of Delta-Notch signaling in cerebral cavernous malformations." Neurosurg Rev.

29. Kar, S., A. Samii and H. Bertalanffy (2015). "PTEN/PI3K/Akt/VEGF signaling and the cross talk to KRIT1, CCM2, and PDCD10 proteins in cerebral cavernous malformations." Neurosurg Rev 38(2): 229-236; discussion 236-227.

30. Khan, H. A., Y. Zhao, L. Wang, Q. Li, Y. A. Du, Y. Dan and L. J. Huo (2015). "Identification of miRNAs during mouse postnatal ovarian development and superovulation." J Ovarian Res 8: 44.

31. Kuehbacher, A., C. Urbich and S. Dimmeler (2008). "Targeting microRNA expression to regulate angiogenesis." Trends Pharmacol Sci 29(1): 12-15.

32. Li, P., M. Shen, F. Gao, J. Wu, J. Zhang, F. Teng and C. Zhang (2016). "An Antagomir to MicroRNA106b-5p Ameliorates Cerebral Ischemia and Reperfusion Injury in Rats Via Inhibiting Apoptosis and Oxidative Stress." Mol Neurobiol.

33. Liao, Y., G. K. Smyth and W. Shi (2014). "featureCounts: an efficient general purpose program for assigning sequence reads to genomic features." Bioinformatics 30(7): 923-930.

34. Liquori, C. L., M. J. Berg, F. Squitieri, T. P. Leedom, L. Ptacek, E. W. Johnson and D. A. Marchuk (2007). "Deletions in CCM2 are a common cause of cerebral cavernous malformations." Am J Hum Genet 80(1): 69-75.

35. Liu, F. J., K. Y. Lim, P. Kaur, S. Sepramaniam, A. Armugam, P. T. Wong and K. Jeyaseelan (2013). "microRNAs Involved in Regulating Spontaneous Recovery in Embolic Stroke Model." PLoS One 8(6): e66393.

36. Liu, J., S. F. Jennings, W. Tong and H. Hong (2011). "Next generation sequencing for profiling expression of miRNAs: technical progress and applications in drug development." J Biomed Sci Eng 4(10): 666-676.

37. Lopez-Ramirez, M. A., A. Reijerkerk, H. E. de Vries and I. A. Romero (2016). "Regulation of brain endothelial barrier function by microRNAs in health and neuroinflammation." FASEB J 30(8): 2662 2672. 
38. Lou, Y. L., F. Guo, F. Liu, F. L. Gao, P. Q. Zhang, X. Niu, S. C. Guo, J. H. Yin, Y. Wang and Z. F. Deng (2012). "miR-210 activates notch signaling pathway in angiogenesis induced by cerebral ischemia." Mol Cell Biochem 370(1-2): 45-51.

39. Love, M. I., W. Huber and S. Anders (2014). "Moderated estimation of fold change and dispersion for RNA-seq data with DESeq2." Genome Biol 15(12): 550.

40. Motameny, S., S. Wolters, P. Nurnberg and B. Schumacher (2010). "Next Generation Sequencing of miRNAs - Strategies, Resources and Methods." Genes (Basel) 1(1): 70-84.

41. Pavlidis, P. and W. S. Noble (2003). "Matrix2png: a utility for visualizing matrix data." Bioinformatics 19(2): 295-296.

42. Renz, M., C. Otten, E. Faurobert, F. Rudolph, Y. Zhu, G. Boulday, J. Duchene, M. Mickoleit, A. C. Dietrich, C. Ramspacher, E. Steed, S. Manet-Dupe, A. Benz, D. Hassel, J. Vermot, J. Huisken, E. Tournier-Lasserve, U. Felbor, U. Sure, C. Albiges-Rizo and S. Abdelilah-Seyfried (2015). "Regulation of beta1 integrin-Klf2-mediated angiogenesis by CCM proteins." Dev Cell 32(2): 181-190.

43. Rerat, K., F. Parker, G. Nasser, D. Vidaud, F. Riant, E. Tournier-Lasserve and C. Denier (2015). "Occurrence of multiple Cerebral Cavernous Malformations in a patient with Neurofibromatosis type 1." J Neurol Sci 350(1-2): 98-100.

44. Riant, F., F. Bergametti, X. Ayrignac, G. Boulday and E. Tournier-Lasserve (2010). "Recent insights into cerebral cavernous malformations: the molecular genetics of CCM." FEBS J 277(5): 1070-1075.

45. Samii, M., R. Eghbal, G. A. Carvalho and C. Matthies (2001). "Surgical management of brainstem cavernomas." J Neurosurg 95(5): 825-832.

46. Schneider, H., M. Errede, N. H. Ulrich, D. Virgintino, K. Frei and H. Bertalanffy (2011). "Impairment of tight junctions and glucose transport in endothelial cells of human cerebral cavernous malformations." J Neuropathol Exp Neurol 70(6): 417-429.

47. Schulz, G. B., E. Wieland, J. Wustehube-Lausch, G. Boulday, I. Moll, E. Tournier-Lasserve and A. Fischer (2015). "Cerebral Cavernous Malformation-1 Protein Controls DLL4-Notch3 Signaling Between the Endothelium and Pericytes." Stroke 46(5): 1337-1343.

48. Seok, J. K., S. H. Lee, M. J. Kim and Y. M. Lee (2014). "MicroRNA-382 induced by HIF-1alpha is an angiogenic miR targeting the tumor suppressor phosphatase and tensin homolog." Nucleic Acids Res 42(12): 8062-8072.

49. Shannon, P., A. Markiel, O. Ozier, N. S. Baliga, J. T. Wang, D. Ramage, N. Amin, B. Schwikowski and T. Ideker (2003). "Cytoscape: a software environment for integrated models of biomolecular interaction networks." Genome Res 13(11): 2498-2504.

50. Suarez, Y. and W. C. Sessa (2009). "MicroRNAs as novel regulators of angiogenesis." Circ Res 104(4): 442-454.

51. Sure, U., S. Freman, O. Bozinov, L. Benes, A. M. Siegel and H. Bertalanffy (2005). "Biological activity of adult cavernous malformations: a study of 56 patients." J Neurosurg 102(2): 342-347.

52. Tan, K. S., A. Armugam, S. Sepramaniam, K. Y. Lim, K. D. Setyowati, C. W. Wang and K. Jeyaseelan (2009). "Expression profile of MicroRNAs in young stroke patients." PLoS One 4(11): e7689.

53. Wang, H. W., H. H. Lo, Y. L. Chiu, S. J. Chang, P. H. Huang, K. H. Liao, C. F. Tasi, C. H. Wu, T. N. Tsai, C. C. Cheng and S. M. Cheng (2014). "Dysregulated miR-361-5p/VEGF axis in the plasma and endothelial progenitor cells of patients with coronary artery disease." PLoS One 9(5): e98070. 
54. Wang, P., Y. Luo, H. Duan, S. Xing, J. Zhang, D. Lu, J. Feng, D. Yang, L. Song and X. Yan (2013). "MicroRNA 329 suppresses angiogenesis by targeting CD146." Mol Cell Biol 33(18): 3689-3699.

55. Wang, S., A. B. Aurora, B. A. Johnson, X. Qi, J. McAnally, J. A. Hill, J. A. Richardson, R. BasselDuby and E. N. Olson (2008). "The endothelial-specific microRNA miR-126 governs vascular integrity and angiogenesis." Dev Cell 15(2): 261-271.

56. Wustehube, J., A. Bartol, S. S. Liebler, R. Brutsch, Y. Zhu, U. Felbor, U. Sure, H. G. Augustin and A. Fischer (2010). "Cerebral cavernous malformation protein CCM1 inhibits sprouting angiogenesis by activating DELTA-NOTCH signaling." Proc Natl Acad Sci U S A 107(28): 12640-12645.

57. Yin, K. J., Z. Deng, H. Huang, M. Hamblin, C. Xie, J. Zhang and Y. E. Chen (2010). "miR-497 regulates neuronal death in mouse brain after transient focal cerebral ischemia." Neurobiol Dis 38(1): 17-26.

58. Yin, K. J., M. Hamblin and Y. E. Chen (2015). "Angiogenesis-regulating microRNAs and Ischemic Stroke." Curr Vasc Pharmacol 13(3): 352-365.

59. You, C., I. E. Sandalcioglu, P. Dammann, U. Felbor, U. Sure and Y. Zhu (2013). "Loss of CCM3 impairs DLL4-Notch signalling: implication in endothelial angiogenesis and in inherited cerebral cavernous malformations." J Cell Mol Med 17(3): 407-418.

60. Yuan, Y., R. Kang, Y. Yu, J. Liu, Y. Zhang, C. Shen, J. Wang, P. Wu, C. Shen and Z. Wang (2016). "Crosstalk between miRNAs and their regulated genes network in stroke." Sci Rep 6: 20429.

61. Zawistowski, J. S., L. Stalheim, M. T. Uhlik, A. N. Abell, B. B. Ancrile, G. L. Johnson and D. A. Marchuk (2005). "CCM1 and CCM2 protein interactions in cell signaling: implications for cerebral cavernous malformations pathogenesis." Hum Mol Genet 14(17): 2521-2531.

62. Zheng, X., C. Xu, A. Di Lorenzo, B. Kleaveland, Z. Zou, C. Seiler, M. Chen, L. Cheng, J. Xiao, J. He, M. A. Pack, W. C. Sessa and M. L. Kahn (2010). "CCM3 signaling through sterile 20-like kinases plays an essential role during zebrafish cardiovascular development and cerebral cavernous malformations." J Clin Invest 120(8): 2795-2804.

\section{Figure Legends}

Fig. 1: Representative MRI image of an intra-axial brainstem cavernoma. Axial T2-weighted MRI scan of a 52-year-old female with a large cavernoma located in the upper Pons (yellow arrow). The patient suffered from severe dizziness, numbness on the left side of the face and hypoesthesiaon the left body side. Images and clinical data were kindly provided by Prof. Helmut Bertalanffy (Vascular Neurosurgery, INI, Hannover).

Fig. 2: The miRNA abundance in CCM. Histogram showing miRNAs $(n=764)$ abundance in CCM $(n=3)$ and normal brain $(n=3)$. The $Y$-axis represents the frequency of the miRNAs while the X-axis represents reads per million counts (RPM) for the six libraries sequenced. Cav1, 2 and 3: CCM; Con1, 2 and 3: Normal brain controls. 
Fig. 3: Volcano plot: CCM versus healthy brains. Volcano plot showing pairwise comparison between CCM and healthy brains. The Y-axis represents the negative of $\log 10$ (FDR corrected padj) and the X-axis corresponds to difference log fold-change. Red and green dots indicates miRNAs significantly up-and downregulated.

Fig. 4: Differential expression profiles of miRNAs in CCM. Heatmap illustrating 52 significantly regulated miRNAs among six libraries (cav1, cav2 and cav3) and (con1, con2 and con3). Color code (scale bar) indicates expression intensities obtained from the sequencing results (red and green corresponds to downregulated and upregulated miRNAs respectively).

Fig. 5: qRTPCR validation of randomly selected miRNAs. The columns represents the downregulated miRNAs expression for let-7b-5p, miR-181a-2-3p and miR-95-3p in CCM ( $\mathrm{n}=3$ ) as compared to control samples. * $\mathrm{p} \leq 0.05, * * \mathrm{p} \leq 0.01$. Y-axis represents fold difference with respect to control while $\mathrm{X}$-axis represents the miRNAs.

Fig. 6: Putative miRNA-mRNA target interaction map. A network map illustrating miRNAs and their CCMrelevant mRNAs. A total of 1877 mRNAs targeted by 5 prioritized miRNAs (let-7b-5p, miR-361-5p, miR-3703p, miR-181a-2-3p and miR-95-3p) were identified using web based application software Cytoscape 3.3.0. The red solid circles represent the miRNAs while the green boxes represent their mRNA targets. The specific interaction between the miRNAs and their target genes are shown by red lines.

Table 1. Table representing patient tissue samples and their clinical characteristics.

Table 2. Subsequent downstream steps applied in the identification of prioritized miRNAs with increasing order of statistical stringency.

Table 3. Identification of experimentally verified mRNAs targeted by each of the 5 prioritized miRNAs.

Additional file 1: Table S1. List of 52 significantly regulated miRNAs expressed in CCM as compared to control samples.

Additional file 2: Table S2. List of putative mRNAs targeted by the five miRNAs identified by miRWalk 2.0. 
Fig. 1

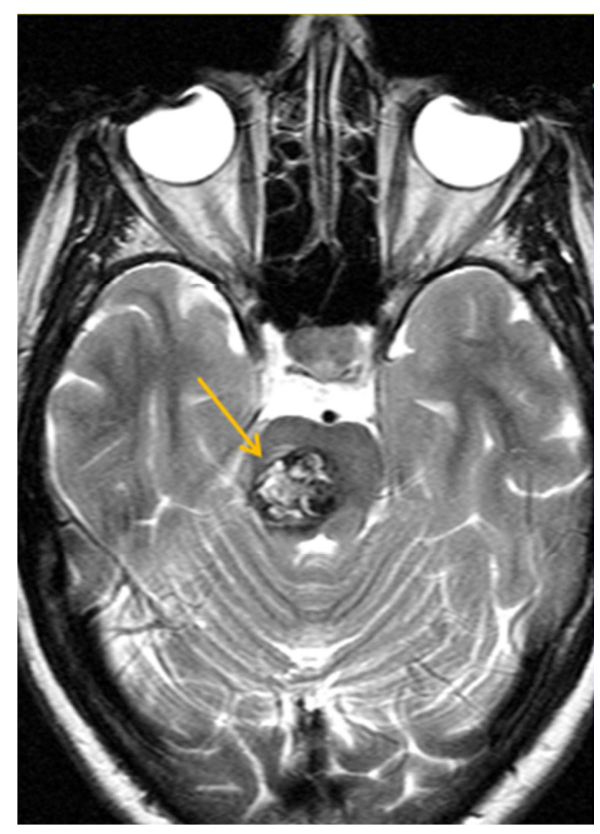

Fig. 2

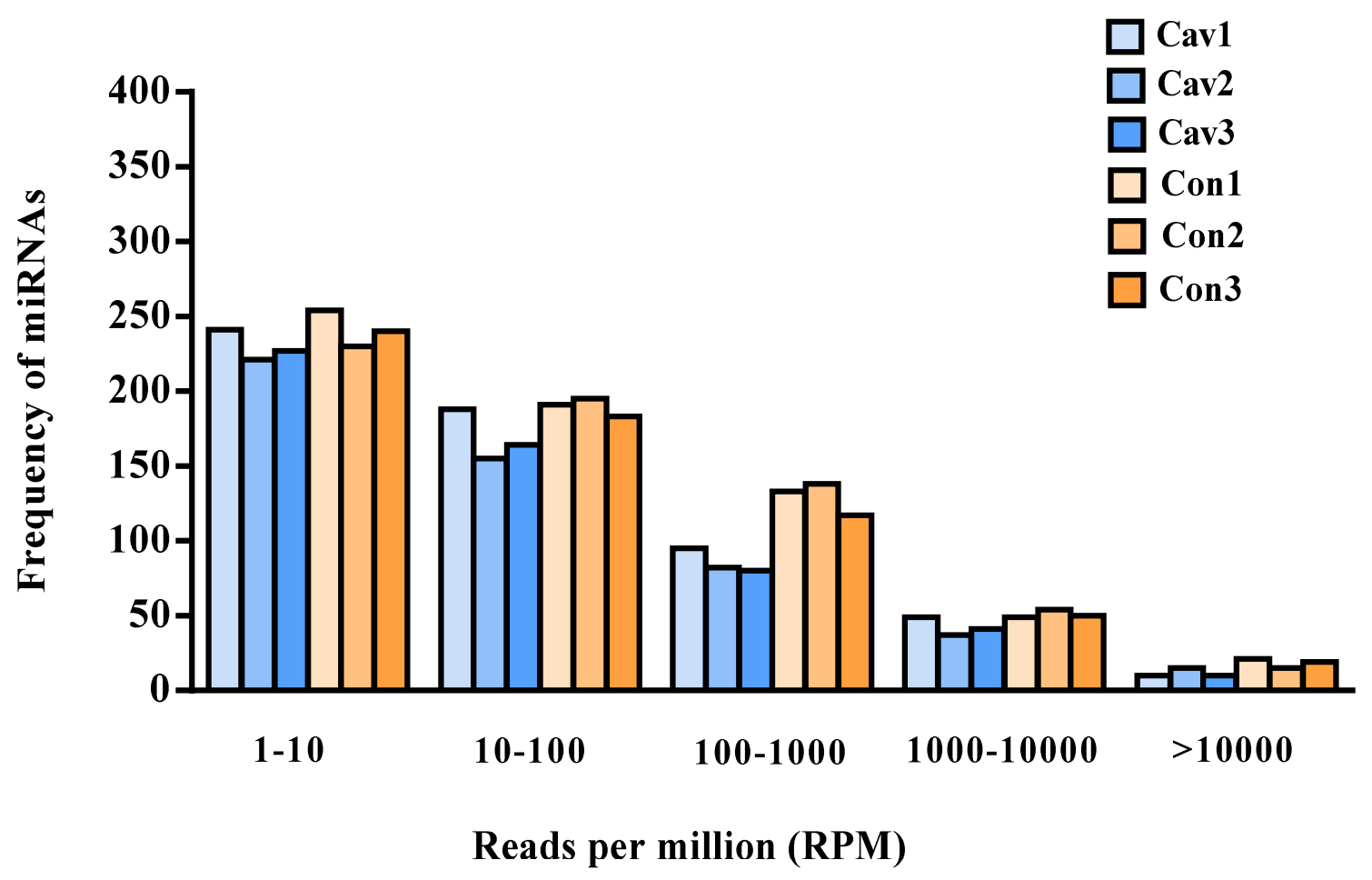


Fig. 3

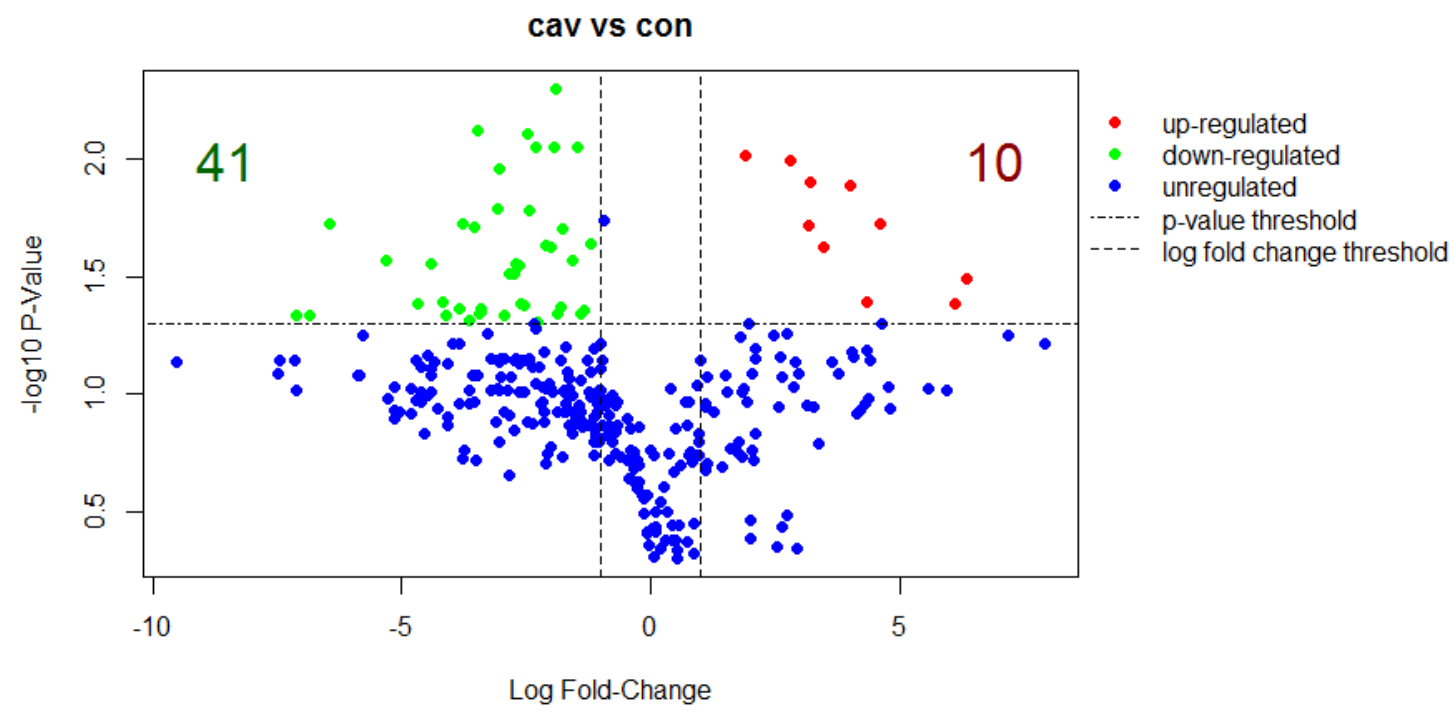


Fig. 4

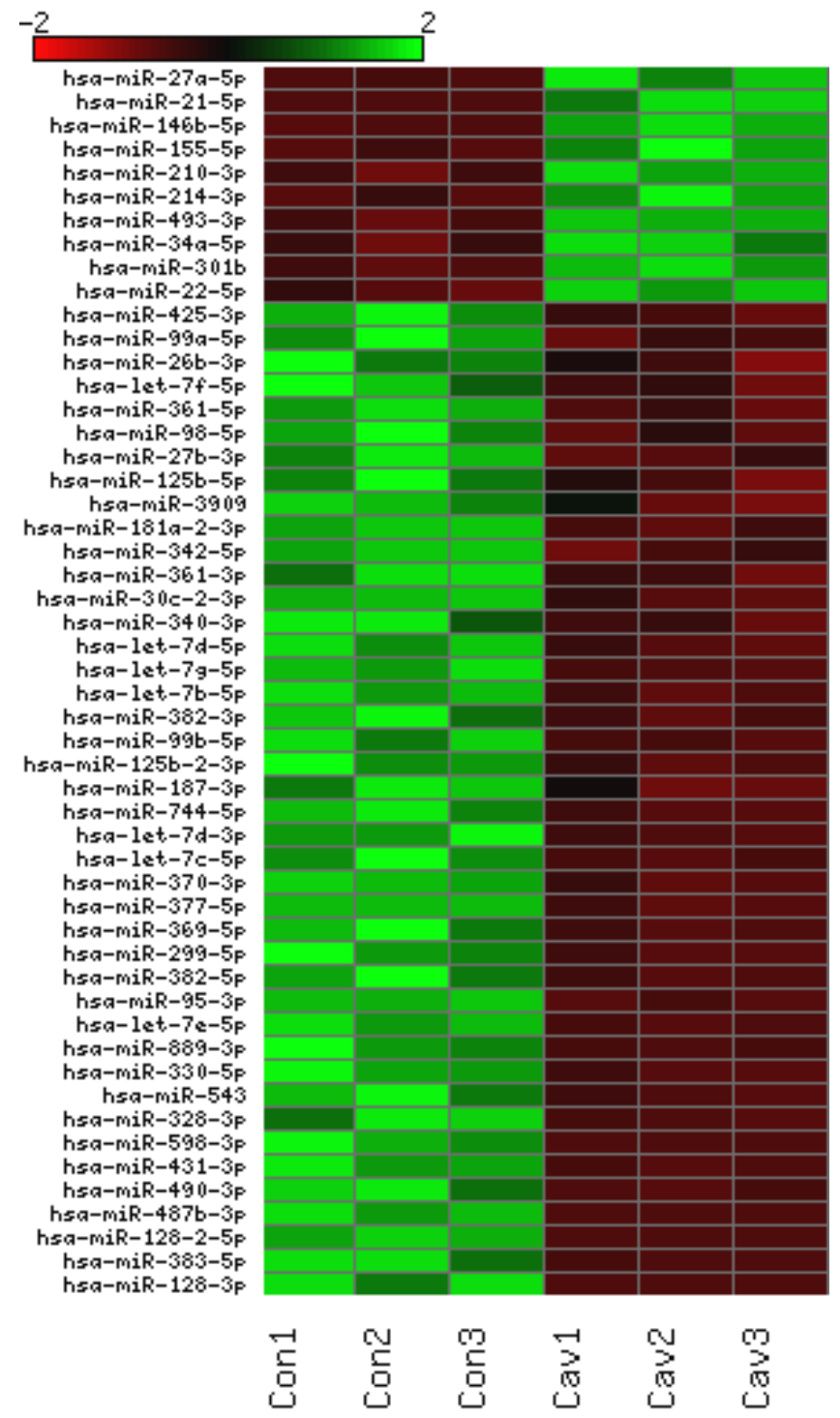


Fig. 5

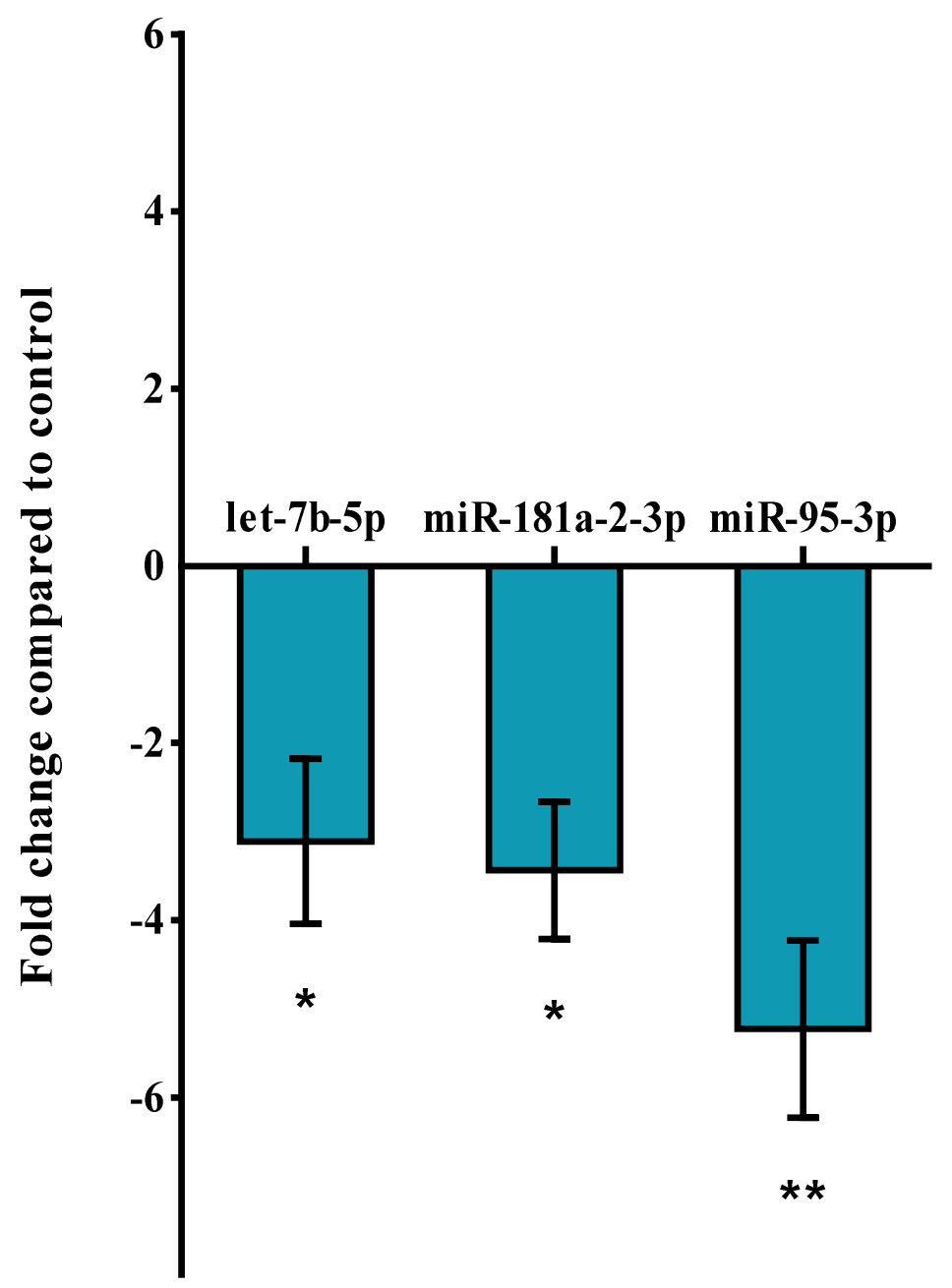


Fig. 6

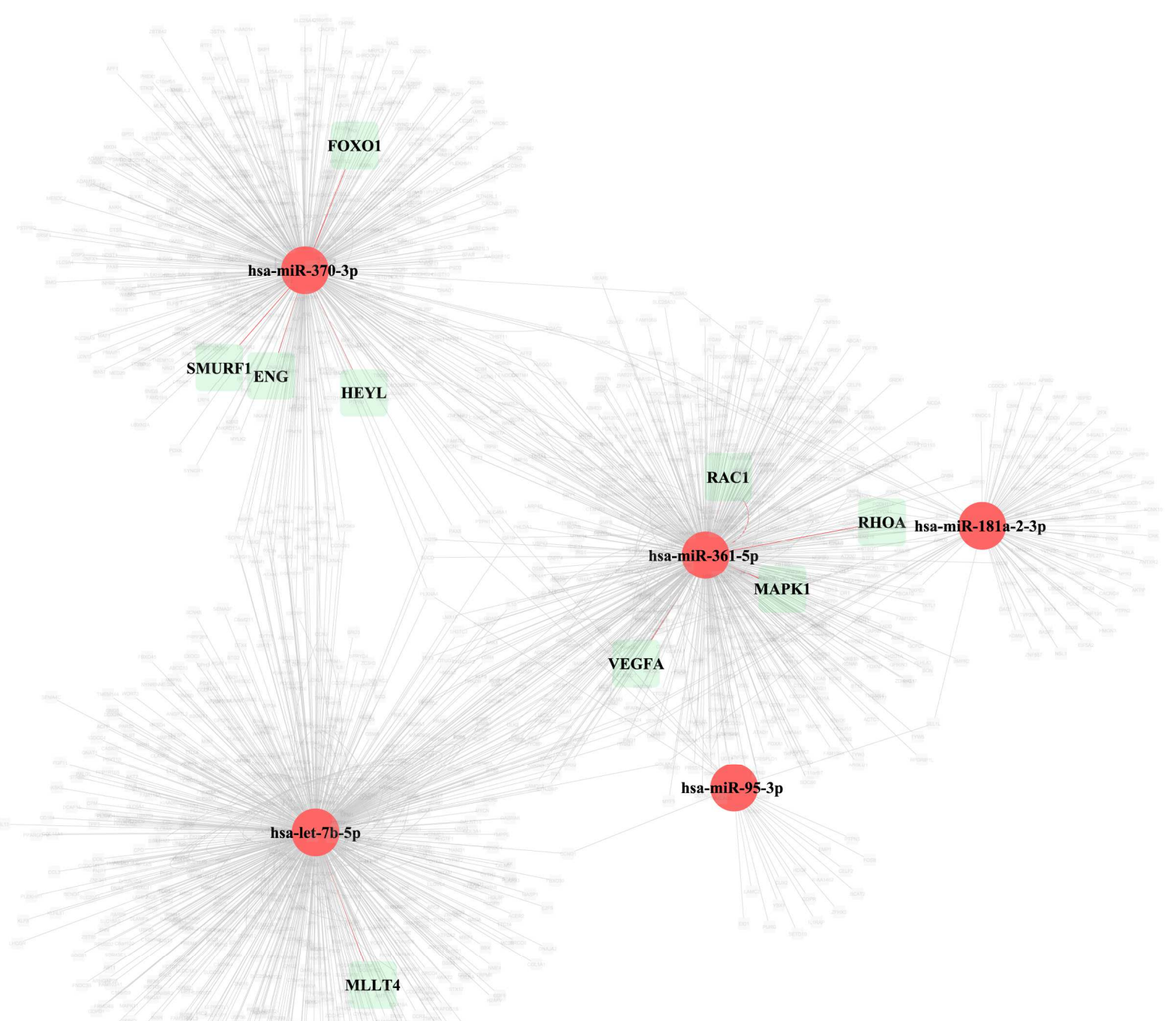




\section{Table 1.}

\begin{tabular}{|c|c|c|c|c|c|c|}
\hline Patient cases & Sex & Age (years) & Lesion Localization & Single/Multiple Lesions & Bleeding episodes & Symptoms \\
\hline Cav1 & M & 36 & Midbrain & $\begin{array}{l}\text { Multiple intracranial } \\
\text { lesions }\end{array}$ & one & $\begin{array}{l}\text { Severe hemiparesis, facial paralysis and hemihypaesthesia } \\
\text { of the left leg }\end{array}$ \\
\hline Cav2 & $\mathrm{F}$ & 51 & Pons & Single & two & Dizziness, double vision and a right-sided sixth nerve palsy \\
\hline Cav2 & $\mathrm{F}$ & 47 & Pons & Single & one & $\begin{array}{l}\text { Severe dizziness, numbness on left face and hypoesthesia } \\
\text { on the left-side of the body }\end{array}$ \\
\hline
\end{tabular}

Table 2.

Increasing order of statistical stringency in CCM tissue samples

\begin{tabular}{|llll|}
\hline Total miRNA sequenced & $\begin{array}{l}\text { RPM } \geq 30 \text { in } \\
\mathbf{2} \text { out of } 6 \text { samples }\end{array}$ & P-value cut-off (0.05) & Benjamini Hochberg (FDR correction) \\
\hline 764 & 327 & 162 & 52 \\
\hline
\end{tabular}

RPM, read per millions, FDR, False Discovery Rate 
Table 3.

\begin{tabular}{|c|c|c|c|c|c|}
\hline microRNAs & $\begin{array}{l}\text { Mean RPM } \\
\text { (CCM/Control) }\end{array}$ & (Adj_P_value_BF) & Regulation (miRNAs) & $\begin{array}{l}\text { No. of validated } \\
\text { mRNA targets }\end{array}$ & CCM-relevant mRNAs \\
\hline hsa-miR-95-3p & 0.089 & 0.007 & Down & 1 & \\
\hline hsa-miR-181a-2-3p & 0.262 & 0.010 & Down & 1 & \\
\hline hsa-let-7b-5p & 0.177 & 0.023 & Down & 981 & $\begin{array}{l}\text { MIB1, HIF1A, PDCD10, TJP1, } \\
\text { OCLN, HES1, MAPK1, ENG }\end{array}$ \\
\hline hsa-miR-370-3p & 0.120 & 0.044 & Down & 4 & NF1 \\
\hline hsa-miR-361-5p & 0.360 & 0.044 & Down & 93 & VEGFA, EGFL7 \\
\hline
\end{tabular}

CCM, cerebral cavernous malformation; RPM, reads per million; BF, Bonferroni correction. 
